\title{
Plasmalogens as a marker of elevated systemic oxidative stress in Parkinson's disease
}

\author{
Charalampos Dragonas ${ }^{1,2}$, Thomas Bertsch ${ }^{3}$, \\ Cornel C. Sieber ${ }^{1,2}$ and Thorolf Brosche ${ }^{2, *}$ \\ ${ }^{1}$ Department of Internal Medicine (Geriatrics), \\ Internal Medicine Clinic II, Clinical Center \\ Nuremberg, Nuremberg, Germany \\ ${ }^{2}$ Institute for Biomedicine of Aging, University of \\ Erlangen-Nuremberg, Nuremberg, Germany \\ ${ }^{3}$ Institute of Clinical Chemistry and Laboratory \\ Medicine, Clinical Center Nuremberg, Nuremberg, \\ Germany
}

Keywords: antioxidant; oxidative stress; Parkinson's disease (PD); plasma; plasmalogens.

Experimental evidence indicates a significant role of oxidative stress in the pathogenesis of idiopathic Parkinson's disease (PD). However, some studies report no decrease in common antioxidants in blood plasma as a marker of oxidative stress (1). The aim of this study was to estimate plasmalogen levels in blood plasma samples of patients with PD, a group of 1-alkenyl-2-acyl-sn-glycero-3-phospholipids considered to be a sensitive surrogate marker of oxidative stress. The precise role played by plasmalogen phospholipids of lipoproteins and cell membranes is not well understood. Considerable attention has been focused on the role of plasmalogens as a source of lipid mediators, based on the activity of calcium-independent phospholipase $A_{2}$ which was shown to catalyze the hydrolysis of arachidonic acid located at the sn2 position of plasmalogens (2). However, plasmalogen status appears to have no influence on stimulated arachidonic acid release in plasmalogendeficient macrophages (3). In addition, plasmalogens can act directly as endogenous antioxidants in defending cell membranes and lipoproteins from reactive radical species. This is accomplished primarily by means of the radical scavenging activity of their vinyl ether bond at the sn 1 position $(2,4-6)$. The present study describes plasmalogen-derived hexadecanal dimethyl acetal (16:0 DMA) concentrations in blood plasma total phospholipid samples from patients with PD, and from age-matched control sub-

*Corresponding author: Dr. T. Brosche, Institute for Biomedicine of Aging, University of Erlangen-Nuremberg, Heimerichstr. 58, 90419 Nuremberg, Germany Phone: + 49-911-3000511, Fax: + 49-911-3000525,

E-mail: t.brosche@aging.med.uni-erlangen.de jects. The vinyl ether bond of plasmalogens and corresponding lyso-compounds was evaluated as a surrogate marker of oxidative stress, which is diminished with increased oxidative imbalance.

The present study included 30 patients with PD diagnosed according to the UK PD Society Brain Bank criteria, and 37 age-matched healthy controls, aged 61 years and older. All study participants were consecutively admitted to our geriatric day hospital and fulfilled the criteria of the SENIEUR protocol. This protocol defines healthy aged individuals for immunogerontological studies by setting clear health guidelines based on clinical and laboratory data (7). Briefly, the following exclusion criteria were applied to all participants: abnormal hematological and hepatic values, current myocardial infarction and stroke, cardiovascular disease, hypertension, diabetes mellitus, hyperuricemia, current infections and inflammation [defined as a serum C-reactive protein (CRP) value $>10 \mathrm{mg} / \mathrm{L}]$, malignancy, malnutrition and alcoholism. We excluded subjects with other neurodegenerative disease, those on special diets, or taking antioxidant supplements, or drugs with known influence on the immune system. Participants with prescribed antiparkinsonian drugs, acetylsalicylic acid (100 $\mathrm{mg}$ ), or statins were included. All PD patients were treated with antiparkinsonian drugs, including levodopa (26 cases), direct dopaminergics (8 cases), amantadine (8 cases), catechol-O-methyl transferase inhibitors (6 cases), and monoamine-oxidase-B-inhibitors ( 4 cases). Reasons for admission to the geriatric day hospital were adjustment of antiparkinsonian medication and physiotherapy in patients with PD, or treatment of depression or chronic pain syndrome in controls. Each participant provided written informed consent. The study protocol was approved by the Ethics Committee of the University of ErlangenNuremberg, Germany.

Fasting peripheral venous blood samples were taken between 9.00 am and 10.00 am. Serum and EDTA plasma samples were obtained following centrifugation at $1800 \times g$ for $15 \mathrm{~min}$, and stored at $-28^{\circ} \mathrm{C}$ until analysis for plasmalogens. Serum albumin was measured nephelometrically using an Immage Nephelometer (Beckman-Coulter Ltd., Krefeld, Germany). The Olympus AU2700 Clinical Chemistry System and manufacturer's reagents (Olympus, Hamburg, Germany) were used to measure cholesterol (CH), high-density lipoprotein cholesterol (HDL-C) and triglyceride (TG) concentrations. CRP concentrations were determined with the Dynamik CRP AU kit (Bio- 
med, Munich, Germany). Low-density lipoprotein cholesterol (LDL-C) was calculated using the Friedwald formula. Ferritin was measured using kits from Roche Diagnostics with the Elecsys 2010 immunoassay system (Roche Diagnostics, Mannheim, Germany). Retinol (vitamin A) and $\alpha$-tocopherol ( $\alpha$-Toc) (vitamin E) concentrations were measured with a commercially available kit (Chromsystems, Munich, Germany) using HPLC (Hewlett Packard Series 1100, Agilent Technologies GmbH, Waldbronn, Germany).

Plasmalogens were measured as described previously (8). Briefly, lipids from lyophilized samples of $0.5 \mathrm{~mL}$ EDTA plasma were extracted and fractionated into lipid classes using silica columns. The isolated total phospholipid fractions were transesterified with borontrifluoride and methanol, yielding mixtures of fatty acid methyl esters (FAME) and plasmalogen derived fatty aldehyde DMA. The resultant mixtures were measured using temperature-programmed capillary gas chromatography on a 50-m WCOT BPX70 column (SGE Deutschland, Darmstadt, Germany). A 5890 series II gas chromatography system (Hewlett Packard) was used, equipped with a temperature-programmed injector (KAS, Gerstel GmbH, Mühlheim/ Ruhr, Germany), a flame ionization detector, a 7683 series autosampler, and a Chem Station B 02.04 software package (Agilent Technologies Inc., Palo Alto, CA, USA). Analyses were performed in triplicate. DMA values are presented as percentage weight of total phospholipid FAME and DMA. Five analyses of a pooled EDTA plasma sample yielded a coefficient of variation (CV) of $2.84 \%$. For all other parameters, the CV was $<5 \%$.

Table 1 summarizes some demographic, clinical and laboratory data for patients with PD and for agematched healthy controls. There are no statistically significant differences between groups for most of the variables. The serum albumin concentrations are slightly lower in patients with PD compared with the control group (Table 1). This difference is possibly due to the high vulnerability of albumin to oxidation. The oxidation products of albumin are a major component of circulating advanced oxidized proteins during inflammatory states. In addition, albumin is a major source of reactive serum thiol goups, and decreased concentrations have been described as an antioxidative marker in the serum of patients with PD.

The lipid normalized $\alpha$-Toc concentrations are $\sim 10 \%$ higher in patients with PD compared to controls (Table 1). Similarly, $\alpha$-Toc concentrations normalized to albumin levels are $12 \%$ higher in the PD group, although not statistically different from controls. These results agree with former reports of no decrease in plasma concentrations of vitamins $E$ and $A$ in PD patients compared with controls (1, and literature cited therein).

Plasmalogen derived 16:0 DMA concentrations of plasma phospholipids in patients with PD are $\sim 12 \%$

Table 1 Demographic, clinical and laboratory data of patients with Parkinson's disease (PD) and of age-matched controls.

\begin{tabular}{|c|c|c|c|}
\hline & $\begin{array}{l}\text { PD patients }{ }^{a} \\
\mathrm{n}=30\end{array}$ & $\begin{array}{l}\text { Controls }^{a} \\
\mathrm{n}=37\end{array}$ & $p$-Value ${ }^{b}$ \\
\hline \multicolumn{4}{|l|}{ Demographic and clinical data } \\
\hline Age, years & $75.7 \pm 6.6$ & $78.2 \pm 8.0$ & 0.17 \\
\hline Gender, $\mathrm{n}$, male/female & $16 / 14$ & $15 / 22$ & 0.33 \\
\hline Statins, $n, \%^{\mathrm{c}}$ & $4,13 \%$ & $11,30 \%$ & 0.14 \\
\hline Acetylsalicylic acid, $100 \mathrm{mg}, \mathrm{n}, \%^{\mathrm{c}}$ & $12,40 \%$ & $18,49 \%$ & 0.62 \\
\hline Smoking, $n, \%^{d}$ & $0,0 \%$ & $2,5.5 \%$ & 0.49 \\
\hline Body mass index, $\mathrm{kg} / \mathrm{m}^{2}$ & $28.4 \pm 5.4$ & $26.5 \pm 4.1$ & 0.11 \\
\hline Duration of PD, years & $5.1 \pm 3$ & n.a. & \\
\hline Stage of $P D^{e}$ & $3.4 \pm 0.8$ & & \\
\hline \multicolumn{4}{|l|}{ Laboratory data } \\
\hline Cholesterol, mmol/L & $5.37 \pm 0.97$ & $5.37 \pm 0.93$ & 0.98 \\
\hline $\mathrm{HDL}-\mathrm{C}, \mathrm{mmol} / \mathrm{L}$ & $1.53 \pm 0.4$ & $1.6 \pm 0.55$ & 0.61 \\
\hline LDL-C, mmol/L & $3.16 \pm 0.8$ & $3.1 \pm 0.87$ & 0.76 \\
\hline $\mathrm{TG}, \mathrm{mmol} / \mathrm{L}$ & $1.49 \pm 0.51$ & $1.5 \pm 0.67$ & 0.93 \\
\hline Albumin, $g / L$ & $39.7 \pm 2.4$ & $41.1 \pm 3.1$ & $0.049^{b}$ \\
\hline Ferritin, $\mathrm{pmol} / \mathrm{L}$ & $436 \pm 677$ & $301 \pm 257$ & 0.27 \\
\hline 16:0 DMA, \% & $0.42 \pm 0.08$ & $0.47 \pm 0.09$ & $0.028^{\mathrm{b}}$ \\
\hline Retinol, $\mu \mathrm{mol} / \mathrm{L}$ & $2.75 \pm 0.81$ & $2.50 \pm 0.62$ & 0.17 \\
\hline Retinol/cholesterol, $\mu \mathrm{mol} / \mathrm{mmol}$ & $0.53 \pm 0.2$ & $0.47 \pm 0.12$ & 0.14 \\
\hline Retinol/cholesterol $+\mathrm{TG}, \mu \mathrm{mol} / \mathrm{mmol}$ & $0.41 \pm 0.13$ & $0.37 \pm 0.08$ & 0.12 \\
\hline Retinol/albumin, $\mu \mathrm{mol} / \mathrm{g}$ & $0.07 \pm 0.02$ & $0.06 \pm 0.01$ & 0.06 \\
\hline$\alpha$-Toc, $\mu \mathrm{mol} / \mathrm{L}$ & $30.6 \pm 6.6$ & $27.7 \pm 8.1$ & 0.12 \\
\hline$\alpha$-Toc/cholesterol, $\mu \mathrm{mol} / \mathrm{mmol}$ & $5.78 \pm 1.13$ & $5.17 \pm 1.19$ & $0.037^{\mathrm{b}}$ \\
\hline$\alpha$-Toc/cholesterol $+\mathrm{TG}, \mu \mathrm{mol} / \mathrm{mmol}$ & $4.47 \pm 0.72$ & $4.02 \pm 0.87$ & $0.027^{\mathrm{b}}$ \\
\hline$\alpha$-Toc/albumin, $\mu \mathrm{mol} / \mathrm{g}$ & $0.77 \pm 0.16$ & $0.68 \pm 0.21$ & 0.053 \\
\hline
\end{tabular}

${ }^{a}$ Data are given as mean $\pm S D$, or as case numbers; ${ }^{b}$ significance level of between group comparison using $\chi^{2}$-test and ANOVA as appropriate; the Mann-Whitney U-test was used to check for differences of plasmalogen-derived 16:0 DMA values; ${ }^{\circ}$ regular drug intake; ${ }^{d}$ smoking of at least one cigarette pack per day; ${ }^{e}$ according to Hoehn and Yahr (9). HDL-C, high-density lipoprotein cholesterol; LDL-C, low-density lipoprotein cholestrol; TG, triglycerides; n.a., not applicable; 16:0 DMA, plasmalogen-derived hexadecanal dimethyl acetal; $\alpha$-Toc, $\alpha$-tocopherol; PD, Parkinson's disease. 
lower than concentrations seen in controls $(p=0.028$, Table 1). This corresponds to a decrease of about $20 \%$ of the plasmalogen portion of plasma total phospholipids. This is because (i) plasmalogen molecules include one fatty acid acyl group, but the majority of plasma glycerophospholipid molecules include two, and (ii) total plasma phospholipid consists of $\sim 80 \%$ of diacylglycerophospholipids. There is considerable overlap of the individual 16:0 DMA values, as can be seen in Figure 1.

The deficiency of plasmalogens in degenerated brain areas of patients with Alzheimer's disease (AD) shows no analogy in the affected brain areas of patients with PD (2). Reduced concentrations of plasmalogens were measured in serum at the initial stage of $A D$, and have been claimed to be a marker of preclinical $A D$ (10). Until now, diminished plasmalogen concentrations in serum or plasma from patients with PD have not been published. Plasma plasmalogen concentrations clearly are not useful as a marker for $\mathrm{PD}$, based on the small difference and overlap of values in PD and controls (Figure 1). However, used as a marker of preclinical $A D$, one should consider reduced concentrations due to $\mathrm{PD}$ in the differential diagnosis.

Apart from being an important factor in the pathogenesis of affected brain areas, oxidative stress in PD may also have a systemic origin. Mitochondrial dysfunction was found in the substantia nigra and peripheral tissues including muscle, platelets and lymphocytes. However, as outlined by Sohmiya et al. (1), some authors report no such changes in patients with PD. In addition, plasma concentrations of common antioxidants such as vitamin E, ascorbic acid, uric acid, carotenoids and coenzyme Q10 were not lower than those in normal subjects. An increased

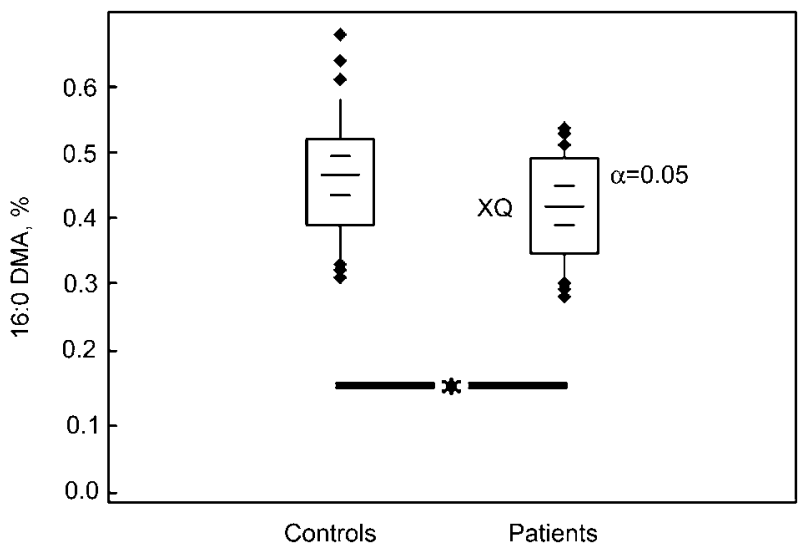

Figure 1 Decreased plasma concentrations of plasmalogens in patients with Parkinson's disease.

Distribution of plasmalogen-derived hexadecanal dimethyl acetal (16:0 DMA) concentrations in EDTA plasma total phospholipids from patients with Parkinson's disease $(n=30)$, compared to age-matched healthy controls $(n=37)$. The box represents the values at the 25th and 75th percentiles; in the box mean (XQ) with confidence interval $(\alpha=0.05)$ is indicated; the vertical lines extend from the 10th to the 90th percentile; all values outside this range are shown as black squares. There were no outliers. percent content of the oxidized form of total coenzyme $\mathrm{Q} 10$ in the plasma of patients with PD has been the first reported confirmatory marker, indicating mild and chronic systemic oxidative stress in PD (1). The reason why this stress is not reflected in the common plasma markers of oxidative stress such as vitamin $\mathrm{E}$ or A has been attributed to different reaction kinetics of different antioxidants observed at early stages of plasma oxidation (1). Our findings showing an $\sim 20 \%$ lower mean concentration of plasmalogens in total phospholipid from plasma of patients with PD, as compared to controls, support the view that mild systemic oxidative stress is a factor in PD pathogenesis. Certainly, not all plasma antioxidants are similar with respect to sensitivity and specificity as markers of oxidative stress.

Another potential confounder to be considered is pharmacological interference. However, there was no significant influence of statin therapy or acetylsalicylic acid use on differences between groups. However, we cannot rule out an effect of antiparkinsonian drugs on plasmalogen values, since all PD patients were treated with these compounds. Whether levodopa treatment raises or decreases plasmalogen concentrations is not known. In sporadic PD, plasma lipid peroxidation was negatively related to the intake of I-dopa, as were free radical concentrations in peripheral blood mononuclear cells. This suggests an antioxidant effect of I-dopa (11). Notably, increased oxidative stress was reported in lymphocytes from untreated patients with PD (12). It would be of interest to confirm our results with greater numbers of patients and in patients not treated pharmacologically.

In summary, mild systemic oxidative stress as a factor in the disease course of PD was suggested by $\sim 20 \%$ lower plasmalogen concentrations in plasma total phospholipids in patients with PD compared to healthy controls. This observation might be due to mitochondrial dysfunction found in cerebral as well as peripheral tissues. Plasma plasmalogen concentrations are not useful as a marker for PD, given the small difference and overlap between values in patients with PD and controls. However, they should be considered in the differential diagnosis of initial stages of AD.

\section{Acknowledgements}

The authors gratefully acknowledge the skilful technical assistance of Mrs. Dipl. Ing. Andrea Hanenberg. This work was supported by a fellowship (CD) from the Robert Bosch Foundation, Stuttgart, Germany.

\section{References}

1. Sohmiya M, Tanaka M, Tak NW, Yanagisawa M, Tanino Y, Suzuki Y, et al. Redox status of plasma coenzyme Q10 indicates elevated systemic oxidative stress in Parkinson's disease. J Neurol Sci 2004;223:161-6.

2. Brites $P$, Waterham HR, Wanders RJ. Functions and biosynthesis of plasmalogens in health and disease. Biochim Biophys Acta 2004;1636:219-31. 
3. Gaposchkin DP, Farber HW, Zoeller RA. On the importance of plasmalogen status in stimulated arachidonic acid release in the macrophage cell line RAW 264.7. Biochim Biophys Acta 2008;1781:213-9.

4. Zoeller RA, Morand $\mathrm{OH}$, Raetz $\mathrm{CH}$. A possible role for plasmalogens in protecting animal cells against photosensitized killing. J Biol Chem 1988;263:11590-6.

5. Brosche T, Platt D. The biological significance of plasmalogens in defense against oxidative damage (minireview). Exp Gerontol 1998;33:363-9.

6. Engelmann B. Plasmalogens: targets for oxidants and major lipophilic antioxidants. Biochem Soc T 2004;32: 147-50.

7. Ershler WB. The value of the SENIEUR protocol: distinction between "ideal aging" and clinical reality. Mech Ageing Dev 2001;122:134-6.

8. Brosche T, Brueckmann M, Haase KK, Sieber C, Bertsch $\mathrm{T}$. Decreased plasmalogen concentration as a surrogate marker of oxidative stress in patients presenting with acute coronary syndromes or supraventricular tachycardias. Clin Chem Lab 2007;45:689-91.

9. Hoehn MM, Yahr MD. Parkinsonism: onset, progression and mortality. Neurology 1967;17:427-42.

10. Goodenowe DB, Cook LL, Liu J, Lu Y, Jayasinghe DA, Ahiahonu PW, et al. Peripheral ethanolamine plasmalogen deficiency: a logical causative factor in Alzheimer's disease and dementia. J Lipid Res 2007;48:2485-98.

11. Prigione A, Begni B, Galbussera A, Beretta S, Brighina $L$, Garofalo R, et al. Oxidative stress in peripheral blood mononuclear cells from patients with Parkinson's disease: negative correlation with levodopa dosage. Neurobiol Dis 2006;23:36-43.

12. Prigione A, Isaias I, Galbussera A, Brighina L, Begni B, Pezzoli G, et al. Increased oxidative stress in Iymphocytes from untreated Parkinson's disease patients. Parkinsonism doi:10.1016/j.parkreldis.2008.05.013. 